variation on echocardiographic measurements. This can be quantified most clearly in the optimisation process, in which genuine small changes in cardiac function (signal) must be detected among potentially large beat-to-beat variation (noise).

Methods and Results In this large study of biological variability, we performed over 2000 echocardigraphic measurements in 12 patients. We performed separate, replicate measurements at a series of interventricular delays of each potential optimisation modality at rest. This included (i) 3D systolic dyssynchrony index, (ii) aortic preejection time, (iii) interventricular mechanical delay, (iv) LVOT VTI and (v) ORS width. The equivalent of 31 optimisations per patient were performed. For single measurements at each setting, agreement between successive optimisations was low, at 39\% for SDI, 41\% for aortic pre-ejection time, $32 \%$ for IVMD, $54 \%$ for LVOT VTI and $58 \%$ for ORS width. Agreement between one method and another, using single replicates, was similarly low, with the average agreement between optima by two methods being only $18 \%$ similar to pure guesswork. The intraclass correlation coefficient was low for all methods at $0.11,0.51,0.30,0.50$ and 0.55 respectively. The intraclass correlation coefficients improved to $0.19,0.63,0.42,0.54$ and 0.66 $(p=0.001)$ when averages of paired measurements were used. To optimise within $20 \mathrm{~ms}$ or $10 \mathrm{~ms}$ of the true optimum, requires a greater number of measurements, as seen in Abstract 116 figure 1, dependant on the intraclass correlation coefficient. The scatter of optima obtained reduced (improved) significantly when using averaged pairs of measurements compared to single measurements from $23 \mathrm{~ms}$ to $18 \mathrm{~ms}$ (3D SDI), $14 \mathrm{~ms}$ to $10 \mathrm{~ms}$ (aortic pre-ejection time), $28 \mathrm{~ms}$ to $22 \mathrm{~ms}$ (IVMD), $21 \mathrm{~ms}$ to $16 \mathrm{~ms}$ (LVOT VTI) and $14 \mathrm{~ms}$ to $10 \mathrm{~ms}$ QRS duration $(\mathrm{p}=0.0002)$.

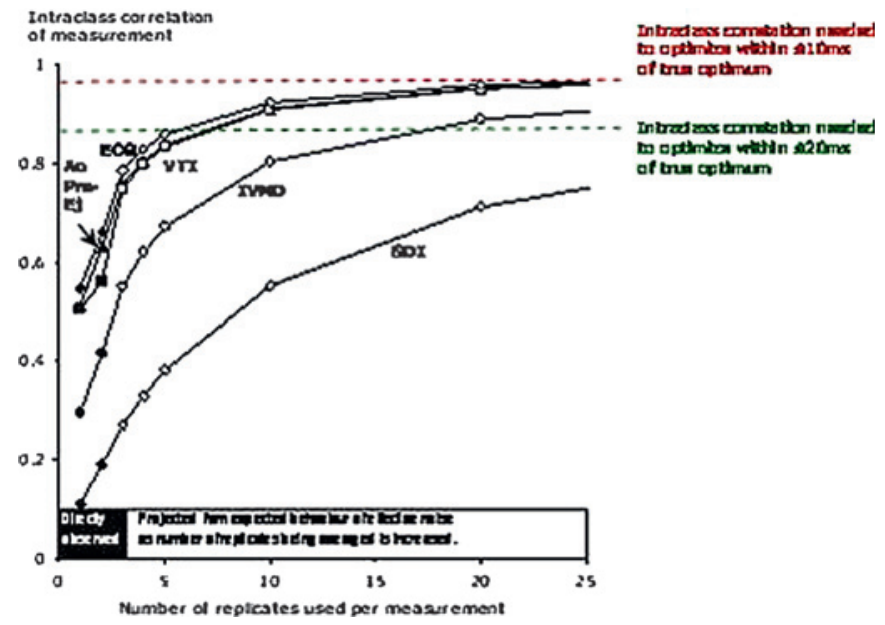

Abstract 116 Figure 1

Conclusions Because of beat-to-beat variability, VV delay optimisation by any of the echocardiographic techniques is not realistic unless multiple replicates are performed and averaged. Smoothing biological variation by averaging multiple measurements allows the full potential of echocardiographic optimisation to be achieved and improves the consistency of optimisation. Trying to save time by performing inadequate numbers of replicates is a false economy and leads to optimisation being a form of randomisation. These observations may also cast light on to why attempts to identify future responders from CRT has not - when tested in externally monitored randomised trials-been fruitful: dyssynchrony assessment to select patients for implantation may need averaging too, and of far more replicate measurements than is current practice. Integration of this biological insight into technological achievements of clinical imaging is necessary, if reliable predictors of which patients will benefit from CRT, are to be developed.

\section{TRICUSPID VALVE ANNULAR DYNAMICS IN NORMAL VS DILATED RIGHT HEARTS; A 3D TOE STUDY}

doi:10.1136/heartjnl-2011-300198.117

L Ring, B Rana, R A Rusk. Papworth Hospital NHS Foundation Trust, Cambridge, UK

Background The tricuspid valve annulus (TVA) is a complex three dimensional structure that is non-planar, and is incompletely understood. The dynamics of the normal TVA has not been described in any significant detail, nor has the impact of abnormal right hearts on the TVA been described. This study was designed to assess the feasibility of assessing the TVA throughout the cardiac cycle using 3D transoesophageal echo (TOE).

Methods 20 patients were included, divided into 2 groups: normal right hearts $(n=10)$, and dilated right hearts $(n=10)$. 3D zoom images of the TVA were acquired using an iE33 imaging platform and X7-2t transducer (Phillips, Andover, Massachusetts, USA). Antero-posterior (AP) diameter, septo-lateral (SL) diameter, area and height were measured at 6 points of the cardiac cycle adapting commercially available software designed for assessing the mitral valve (MVQ, Phillips). The eccentricity ratio was calculated as $\mathrm{AP} / \mathrm{SL}$.

Results TVA area decreases during systole in both groups, and is greatest in mid-diastole. The area is significantly larger in the abnormal group (mean $1795 \mathrm{~mm}^{2}$ abnormal vs $1204 \mathrm{~mm}^{2}$ normal; $\mathrm{p}<0.01$ ). The SL diameter increased more in the abnormal group, resulting in a circular orifice and lower eccentricity ratio throughout the cycle (mean 0.91 abnormal v 1.22 normal; $p<0.01$, see graph). Annular height is similar in both groups but has an upward trend in systole in normals and reduces in abnormals, reaching significance at end systole ( $6.7 \mathrm{~mm}$ vs $4.9 \mathrm{~mm}$; $\mathrm{p}=0.046$ ).

Conclusions In patients with abnormal right hearts, the TVA dilates in a septo-lateral direction, resulting in a more circular orifice. The dynamic changes of the TVA are similar in dilated vs normal right hearts, with the exception of annular height. This pilot study suggests that 3D TOE provides insight into understanding tricuspid annular dynamics.

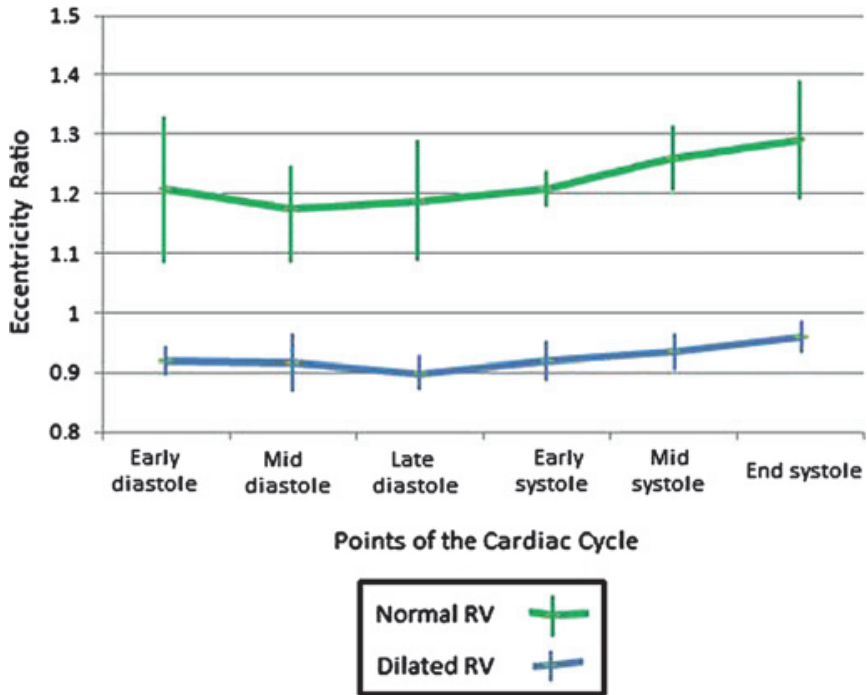

Abstract 117 Figure 1 Eccentricity ratio of the tricuspid valve annulus during the cardiac cycle: normal vs dilated rated hearts. 